\title{
1 Copper selects for siderophore-mediated virulence in Pseudomonas aeruginosa
}

2

3 Luke Lear $^{1 *}$, Elze Hesse ${ }^{2}$, Angus Buckling ${ }^{2}$ and Michiel $\operatorname{Vos}^{1}$.

4

$5 \quad{ }^{1}$ European Centre for Environment and Human Health, University of Exeter Medical School,

6 Penryn, Cornwall, United Kingdom

7

8

9

${ }^{2}$ College of Life and Environmental Science, University of Exeter, Penryn, Cornwall, United Kingdom

*Corresponding author

Keywords: evolution of virulence, opportunistic pathogen, metal detoxification, pyoverdine, coincidental selection.

$\underline{\text { Abstract }}$

Iron is essential for almost all bacterial pathogens and consequently it is actively withheld by their hosts. The production of extracellular siderophores however enables iron sequestration by pathogens, increasing their virulence. Another function of siderophores is extracellular detoxification of non-ferrous metals. Here, we experimentally link the detoxification and virulence roles of siderophores by testing whether the opportunistic pathogen Pseudomonas aeruginosa displays greater virulence after exposure to copper stress. We incubated $P$. aeruginosa under different copper regimes for either two or twelve days. Subsequent growth in a copper-free environment removed phenotypic effects, before quantification of pyoverdine production ( $P$. aeruginosa's primary siderophore) and virulence using the Galleria mellonella infection model. Copper selected for increased pyoverdine production, which was positively associated with virulence. This effect increased with time. We here show a direct link between metal stress and bacterial virulence, highlighting another dimension of the detrimental effects of metal pollution on human health. 
Iron is essential for the growth of almost all bacteria as it serves as a cofactor for many enzymes $(1,2)$. However, as iron most commonly exists in the insoluble form $\mathrm{Fe}^{3+}$ it is of relatively low bioavailability in the majority of environments (1-7). It is therefore essential for bacteria to actively sequester $\mathrm{Fe}^{3+}$ from the environment $(8,9)$, with many bacteria producing iron-chelating siderophore compounds to do this (10). Siderophores aid iron recovery by forming extracellular complexes with $\mathrm{Fe}^{3+}$ which are then taken up by the cell and reduced to the bioavailable form $\mathrm{Fe}^{2+}(11)$. The extracellular nature of siderophores means they can benefit the group around the producer, with only the producer paying the cost of production. As a result siderophore production is an altruistic cooperative trait subject to social evolution (12). Therefore as increased production evolves under iron limitation to benefit the producer and its kin, genotypes with reduced production can also evolve to exploit these producers and gain a fitness advantage (12). Due to the importance of iron for microbial growth, one of the first lines of host defence is to withhold iron from invading pathogens (13). This often involves the production of molecules such as transferrin that bind to iron with very high association constants $(6,13-15)$. Such nutritional immunity can be overcome by pathogens through the production of siderophores, as their very high iron affinity enables 'stealing' iron from the host (15). Siderophores are therefore an important virulence factor in both gram-negative- (16-18) and gram-positive human pathogens $(19,20)$.

In addition to iron, siderophores are known to bind to a range of potentially toxic metals (21). Crucially, these complexes cannot passively diffuse into the cell and consequently the metal is detoxified extracellularly (22). As a result, siderophore production has been shown to increase in the presence of toxic levels of a wide range of non-ferrous metals $(2,3,8,9,21)$. As many of the metal ions that can be bound by siderophores are essential in small amounts, siderophore production is adjusted in response to metal bioavailability (23), with either active uptake of metal-siderophore complexes or other metal chelating compounds used to acquire these micronutrients when they are scarce $(7,24)$. Adjustments in production can be due to both genetic and phenotypic changes, with genetic mutations dictating the limits of siderophore production and phenotypic changes used to regulate production within those limits (25). Although the upper limit of production initially increases with metal toxicity, eventually a threshold is reached as the rising metabolic cost of siderophore production can result in selection for reduced production (8) - analogously to the evolutionary response to iron 
deficiency (26). This cost is further exacerbated when competitors of the producer benefit from the siderophore (12). Consequently when there is strong selection for siderophore production, such as when toxic metals are at high concentrations, per capita siderophore production can be reduced $(8,26,27)$. Phenotypic changes in production occur quickly in response to alterations in the environment, including the abundance of metals and population densities, and allow the cost of production to be minimised $(22,27)$.

Despite strong evidence both for siderophores playing an important role in metal detoxification $(3,8,21,23)$ and for siderophore production being an important determinant for bacterial virulence $(15,28,29)$, whether the presence of toxic metals in non-host environments affects virulence remains untested. Here we aim to experimentally link the dual roles of siderophores by testing the effects of toxic copper on the virulence of the opportunistic human pathogen $P$. aeruginosa. P. aeruginosa is of clinical significance capable of surviving in a range of environments including soil, water and fomites in hospitals $(30,31)$. In clinical settings it is frequently responsible for severe and fatal infections of patients with cystic fibrosis (32), burns (33), and immunosuppressive illnesses (34).

The production of the main $P$. aeruginosa siderophore, pyoverdine, has been shown to both increase under copper stress $(21)$ and to be a virulence factor $(28,35)$. It has also been shown to rapidly evolve (36). We therefore test virulence after both two days ( $\sim 7$ generations) and twelve days ( 40 generations) $(12,37)$. To allow us to exclude phenotypic changes to production, at each timepoint (two and twelve days) populations are transferred to a common garden environment (copper free media) for 24 hours prior to assays. By quantifying pyoverdine production in every population before the virulence assay we are able to test whether its production is associated with virulence. Moreover, we test if this association is the same across two environmentally relevant copper concentrations that span those found in in agricultural soil (38). This paper therefore provides novel data on the role of metal pollution on bacterial virulence.

\section{Methods}

\section{Experimental design}

Pseudomonas aeruginosa PAO1 (39) was grown shaking for 24 hours in glass microcosms containing $6 \mathrm{~mL}$ of King's medium B (KB; 10g glycerol, 20g proteose peptone no. 3, $1.5 \mathrm{~g}$ 
$\mathrm{K}_{2} \mathrm{HPO}_{4}, 1.5 \mathrm{~g} \mathrm{MgSO}_{4}$, per litre) at $28^{\circ} \mathrm{C}$. After homogenisation by vortexing, $60 \mu \mathrm{L}$ was added to 18 microcosms containing $\mathrm{KB}$ mixed with sterilised copper sulphate $\left(\mathrm{CuSO}_{4}\right.$; Alfa Aesar, Massachusetts, United States) to final concentrations of either $0.0,0.1$ or $1.0 \mathrm{~g} / \mathrm{L}$. Inoculated microcosms were kept static at $28^{\circ} \mathrm{C}$ for 48 hours before being thoroughly homogenised and $60 \mu \mathrm{L}$ (1\% by volume) transferred into fresh media (Fig.1). Transfers occurred every two days for twelve days. To control for the physiological effects of copper stress, on days two and twelve all microcosms were homogenised and $60 \mu \mathrm{L}$ transferred into a common garden environment (KB medium without copper). These cultures were grown for 24 hours before pyoverdine production was quantified and aliquots were frozen at $-80^{\circ} \mathrm{C}$ in glycerol at a final concentration of $25 \%$ for virulence assays and to quantify density.

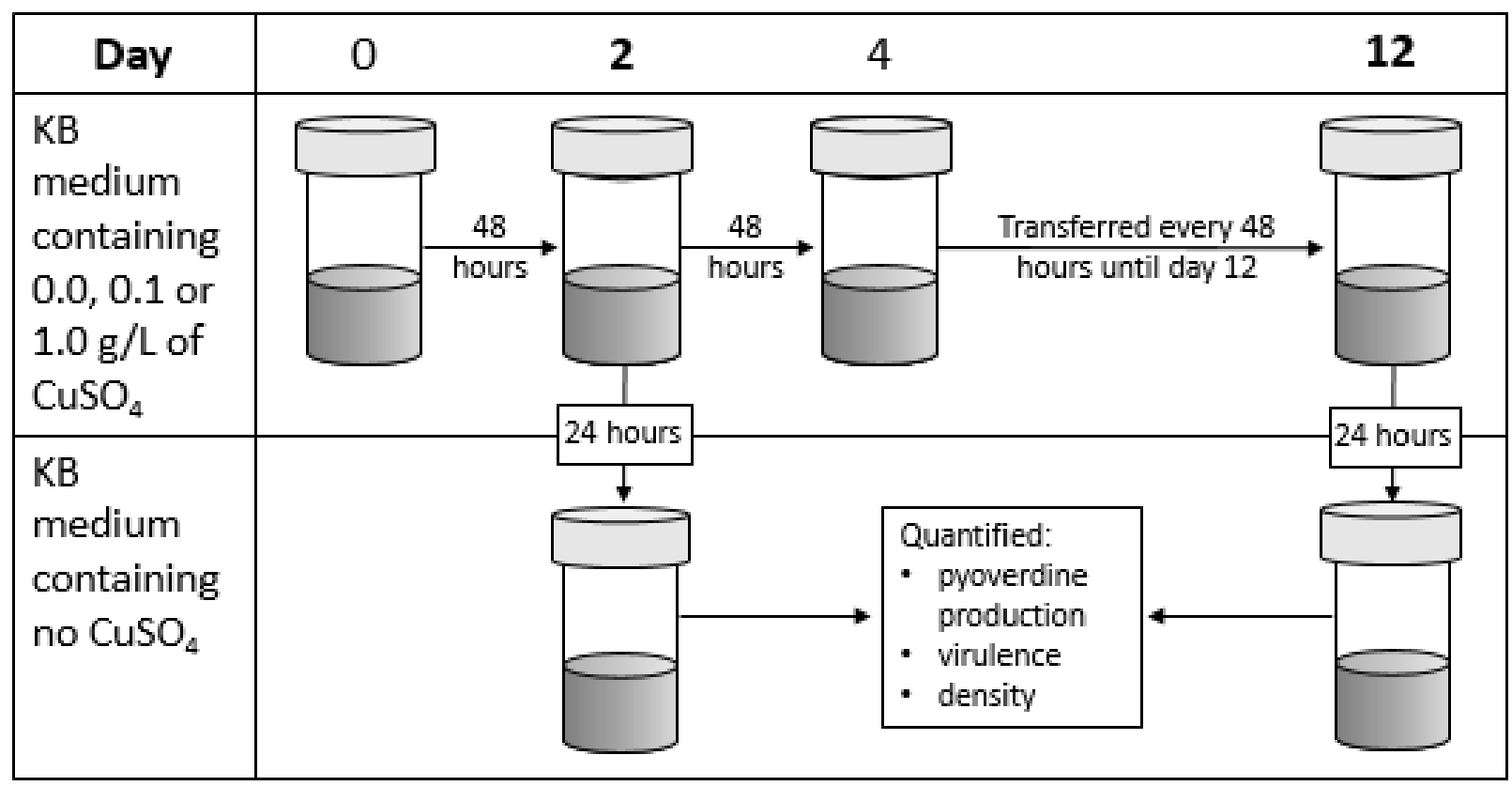

113 Figure 1 Schematic of the experimental design used to test whether copper selects for 114 siderophore-mediated virulence. Microcosms ( $\mathrm{n}=6$ per treatment) containing KB medium at a concentration of either $0.0,0.1$ or $1.0 \mathrm{~g} / \mathrm{L}$ of copper sulphate $\left(\mathrm{CuSO}_{4}\right)$ were inoculated with Pseudomonas aeruginosa, incubated at $28^{\circ} \mathrm{C}$ and transferred every two days into fresh media.

117 On days two and twelve, cultures were transferred into copper free medium for 24 hours before 118 being homogenised, their per capita pyoverdine production quantified and frozen in glycerol at a final concentration of $25 \%$. Virulence and density assays were performed using defrosted samples. 
123 Pyoverdine production was quantified after both two and twelve days. Following 24 hrs in a

124 common garden environment, cultures were thoroughly homogenised before $600 \mu \mathrm{L}$ was 125 transferred into a 96 well-plate $(200 \mu \mathrm{L}$ into three separate wells). The fluorescence at $460 \mathrm{~nm}$

126 following excitation at $400 \mathrm{~nm}$ of each of the cultures in the 96-well plate was measured using 127 a BioTek Synergy 2 plate reader (BioTek, Vermont, U.S.A.). Pyoverdine, which fluoresces 128 green, is the only culture component quantified using these excitation and emission parameters, 129 with non-producers giving a zero reading (4). Each culture was measured three times (one 130 reading of each of three wells per culture) and the average of three sterile media readings 131 (containing the relevant copper concentration) was used as a reference. The optical density 132 measurements were used to estimate pyoverdine production per cell using: standardised 133 fluorescence units / $\mathrm{OD}_{600}$. The average of the three technical replicates was used in the 134 analysis.

\section{Galleria mellonella virulence assay}

137 To quantify virulence, we used the insect infection model Galleria mellonella (40). Briefly, 138 defrosted samples were diluted $10^{5}$-fold in $\mathrm{M} 9$ salt buffer $\left(3 \mathrm{~g} \mathrm{KH}_{2} \mathrm{PO}_{4}, 6 \mathrm{~g} \mathrm{Na} 2 \mathrm{HPO}_{4}, 5 \mathrm{~g} \mathrm{NaCl}\right.$ 139 per litre) before $10 \mu \mathrm{L}$ was injected into 20 final instar larvae each per replicate using a $50 \mu \mathrm{L}$ 140 Hamilton syringe (Hamilton, Nevada, USA). Larvae were then incubated at $37^{\circ} \mathrm{C}$ and mortality 141 checked 18 hours post-injection. Larvae were classed as dead when mechanical stimulation of 142 the head caused no response (41). M9-injected and non-injected controls were used to confirm 143 mortality was not due to injection trauma or background G. mellonella mortality; >10\% control 144 death was the threshold for re-injecting (no occurrences).

\section{Quantifying Pseudomonas aeruginosa density}

147 The density of $P$. aeruginosa in the common garden environment was quantified by plating onto agar. To do this, samples were defrosted, serially diluted with M9 salt buffer and plated onto $\mathrm{KB}$ agar. After 48 hours incubation at $28^{\circ} \mathrm{C}$ colonies were counted and the number of colony forming units (CFU) standardised to CFUs per $\mathrm{mL}$.

\section{$\underline{\text { Statistical analysis }}$}

153 The effect of copper on per capita pyoverdine production (standardised fluorescence units / $\left.154 \mathrm{OD}_{600}\right)$ and density $(\mathrm{CFU} / \mathrm{mL})$ of $P$. aeruginosa populations was tested using linear mixed 155 effects models (LMEM) with copper and time as explanatory variables (both factors), as well 
as their 2-way interaction. To determine how copper affected virulence we used a binomial generalised linear mixed model (GLMM), with number of G. mellonella dead versus alive as binomial response variable, and copper and time as explanatory variables, as well as their twoway interaction. To analyse the combined effect of pyoverdine production and density on virulence, we used a similar framework and included pyoverdine production, total CFU and time, along with a two-way pyoverdine-time interaction, as explanatory variables. In all analyses, pyoverdine production and population density were $\log _{10}$-transformed to improve normality, and random intercepts were fitted for individual replicates to account for nonindependency of observations over time.

For all analyses, we used the DHARMa package (42) to check residual behaviour, after which the most parsimonious model was arrived at by sequentially deleting terms and comparing model fits using $\chi$-tests where appropriate. These were followed by Tukey post hoc comparisons with the false detection rate due to multiple comparisons controlled for using the Benjamini and Hochberg method. All analyses were carried out in R version 3.3.3 (43), with the lme4 package used for the LMEMs and GLMMs (44).

\section{$\underline{\text { Results }}$}

\section{$\underline{\text { Toxic copper selects for increased pyoverdine production }}$}

Here we tested the evolutionary consequences of copper stress on Pseudomonas aeruginosa by incubating it with copper for either two or twelve days, and then without copper for one day. All results shown here therefore display the evolutionary consequences of copper stress, as the phenotypic effects are removed by the copper-free common garden environment step. In line with previous findings (3), pyoverdine production evolved to be significantly greater as a function of copper concentration (copper main effect: $X^{2}=35.4$, d.f. $=2, p<0.001$ ). This effect was consistent across the two and twelve day treatments; however production was significantly greater after twelve days compared to two days (time main effect: $X^{2}=161$, d.f. $=1$, $p<0.001$; Fig. 2). Pyoverdine production was significantly higher in the high copper treatment compared to both the control (two days: $\mathrm{p}<0.001$; twelve days: $\mathrm{p}<0.001$ ) and the low copper treatment (two days: $\mathrm{p}=0.032$; twelve days: $\mathrm{p}=0.032$ ). Likewise, the low copper treatment was always significantly higher than the control (two days: $\mathrm{p}=0.004$; twelve days: $\mathrm{p}=0.004$ ). The nonindependence of observations between day two and day twelve samples accounted for only a small amount of the total variation $(\mathrm{SD}=0.029)$ in this model. 


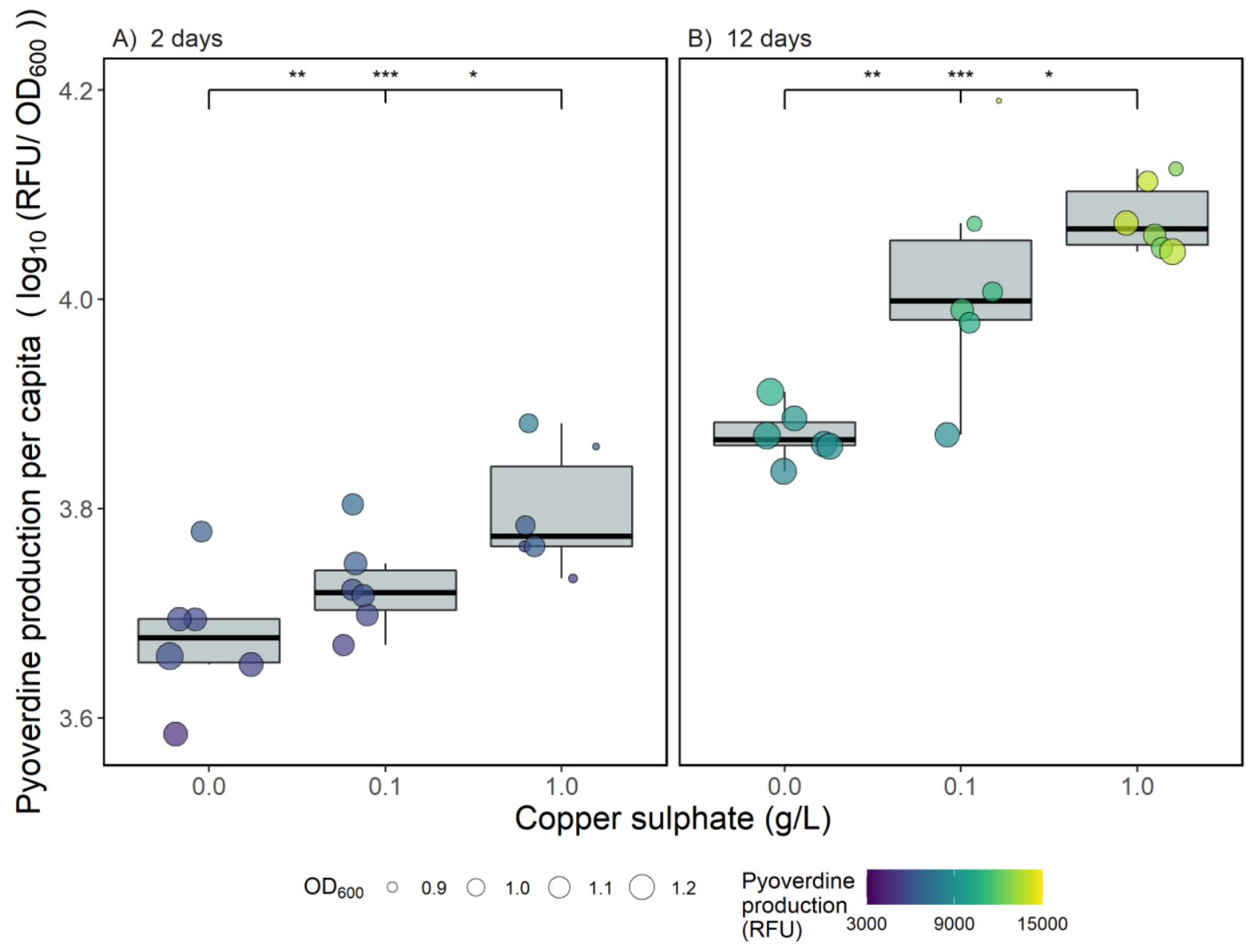

190 Figure 2 Per capita pyoverdine production $\left(\log _{10}\right.$-transformed standardised fluorescence units 191 per $\mathrm{OD}_{600}$ ) by Pseudomonas aeruginosa populations after growth in different concentrations 192 of copper for (A) two days + one day in the absence of copper, or (B) twelve days + one day 193 in the absence of copper. Circles show individual replicates $(n=6)$, with colour indicating total pyoverdine production (standardised fluorescence units) and size showing optical density at $600 \mathrm{~nm}$. Asterisks indicate significant differences between groups $(* * *=0.001, * *=0.01, *=$ 0.05 , NS = non-significant), with the left value comparing the control to the low copper treatment, the middle value comparing the control to the high copper treatment and the right value comparing the low and high copper treatments.

Virulence is higher in populations exposed to copper and this effect increases with time

201 To test whether copper stress caused differences in $P$. aeruginosa virulence, populations were assayed using the Galleria mellonella virulence model. Copper significantly increased virulence (copper main effect: $X^{2}=20.8$, d.f. $=2$, $\mathrm{p}<0.001$ ) with the degree to which increasing in time (copper-time interaction: $X^{2}=6.90$, d.f.=2, $\mathrm{p}=0.031$; Fig. 3). After two days, virulence was significantly higher in the two copper treatments compared to the control $(\mathrm{p}<0.001$ for both comparisons) but these treatments did not differ themselves $(\mathrm{p}=0.34)$. After twelve days 


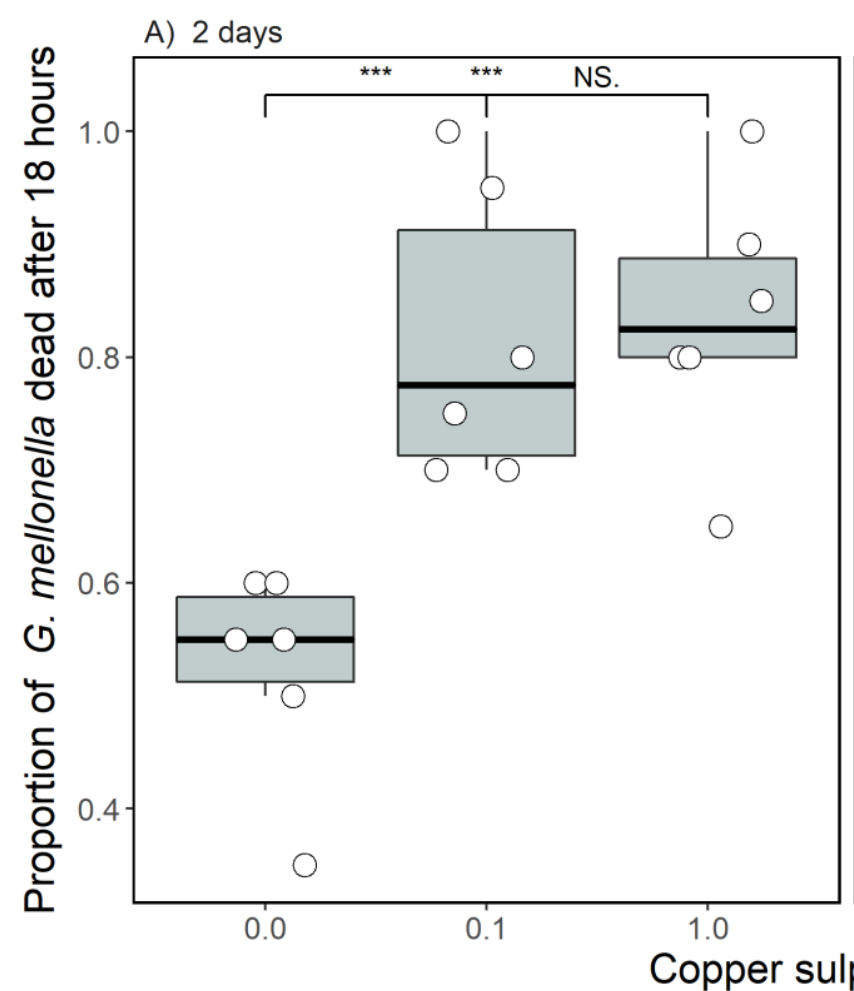

B) 12 days

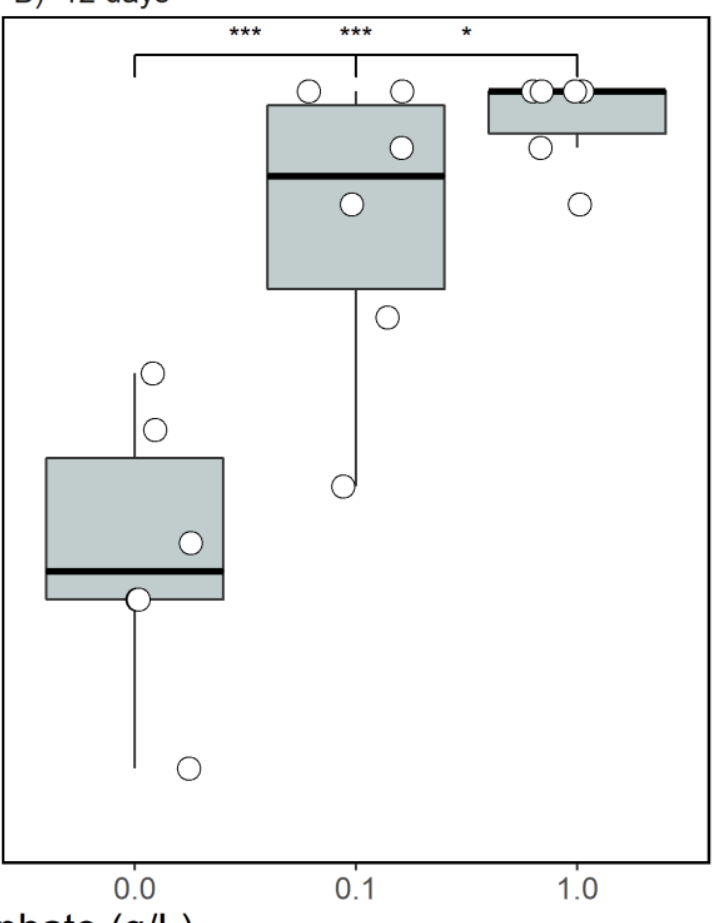

214 Figure 3 The proportion of Galleria mellonella dead 18 hours after being injected with 215 Pseudomonas aeruginosa grown in the presence or absence of copper for (A) two days (+ one 216 day in the absence of copper) or (B) twelve days (+ one day in the absence of copper). $20 \mathrm{G}$. 217 mellonella were injected per replicate (circles show individual replicates; $\mathrm{n}=6$ per unique 218 treatment combination). Asterisks indicate significant differences between groups $(* * *=$ $2190.001, * *=0.01, *=0.05, \mathrm{NS}=$ non-significant), with the left value comparing the control to 220 the low copper treatment, the middle value comparing the control to the high copper treatment 221 and the right value comparing the low and high copper treatments.

\section{The effect of copper on population density differs over time}

224 The effect of copper on population density differed as function of time (copper-time 225 interaction: $X^{2}=20.5$, d.f.=2, $\mathrm{p}<0.001$; Fig. 4). After two days, population densities did not significantly differ between any copper treatment ( $\mathrm{p}=>0.060$ for all contrasts), whereas after 
twelve days of growth density was significantly lower in the two copper treatments compared to the control (high copper - control: $\mathrm{p}=0.013$ and low copper - control: $\mathrm{p}=0.002$ ) but did not differ themselves $(\mathrm{p}=0.48)$. The effect of measuring the same populations at two time points did not explain any of the overall variation in this model $(\mathrm{SD}=0.0)$.

A) 2 days

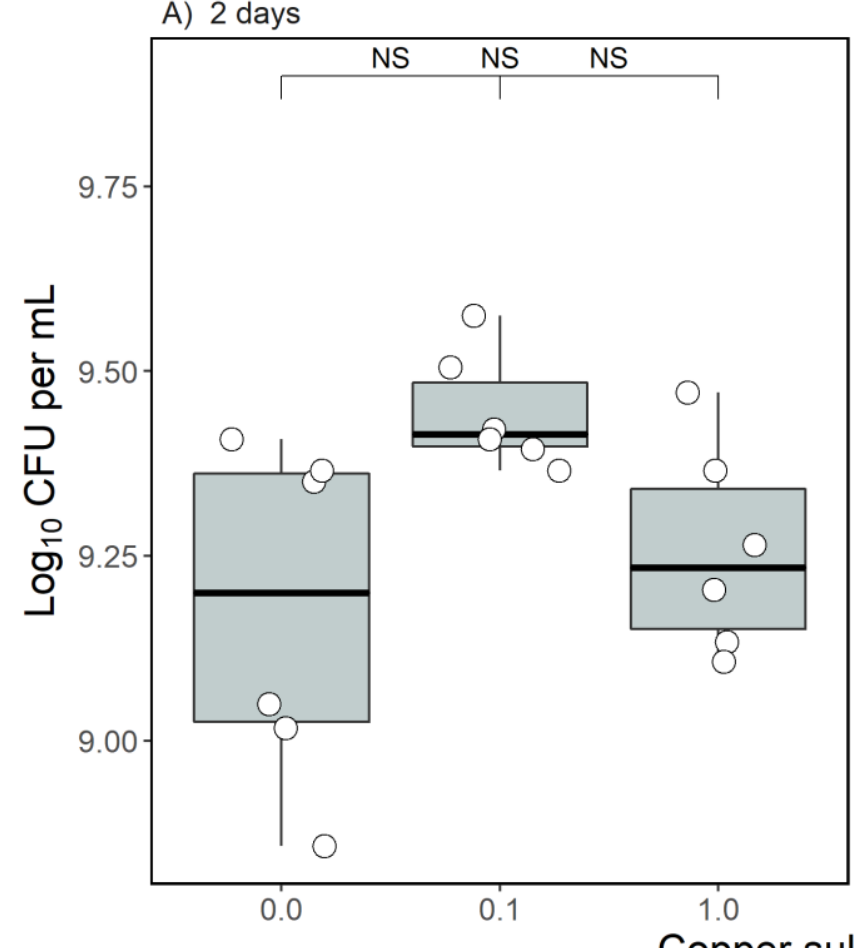

B) 12 days

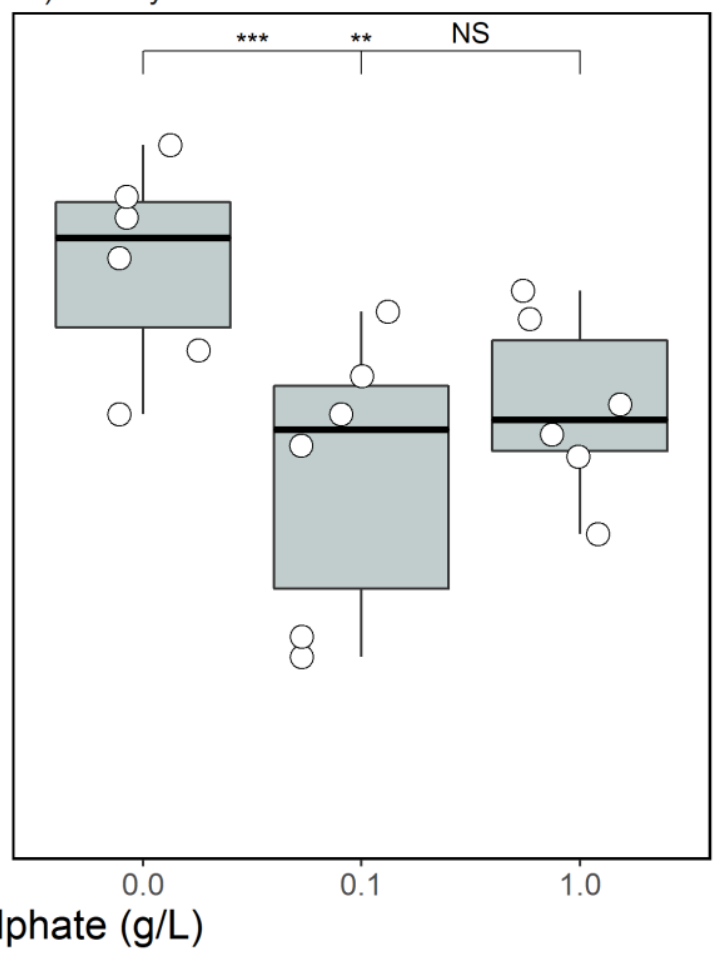

232

233

234

235

236

237

238

239

240

241

242

\section{Virulence is associated with increased pyoverdine production rather than pathogen load}

Figure 4 The density $\left(\log _{10} \mathrm{CFU} \mathrm{mL} \mathrm{mL}^{-1}\right)$ of Pseudomonas aeruginosa populations incubated with copper for two days + one day without copper (panel A) or twelve days + one day without copper (panel B). Asterisks indicate significant differences between groups $(* * *=0.001, * *=$ $0.01, *=0.05, \mathrm{NS}=$ non-significant), with the left value comparing the control to the low copper treatment, the middle value comparing the control to the high copper treatment and the right value comparing the low and high copper treatments.

Finally, we tested whether increased virulence was associated with increased pyoverdine production by replacing 'copper' as an explanatory variable in our GLMM with per capita pyoverdine production and included density as a covariate in this model. Despite there being substantial variation across populations $(\mathrm{SD}=0.86)$ in virulence, virulence increased as a function of pyoverdine production (pyoverdine main effect: $X^{2}=13.9$, d.f. $=1$, p $<0.001$; Fig. 5), 
and this effect did not change with time (pyoverdine-time interaction: $X^{2}=0.009$, d.f. $=1$, $\mathrm{p}=0.93$ ) although virulence was higher after twelve days (time main effect: $X^{2}=5.40$, d.f.=1, $\mathrm{p}=0.02$ ). Population density had no significant effect on virulence (main effect of density in GLMM: $X^{2}=1.09$, d.f.=2, $\left.\mathrm{p}=0.30\right)$.

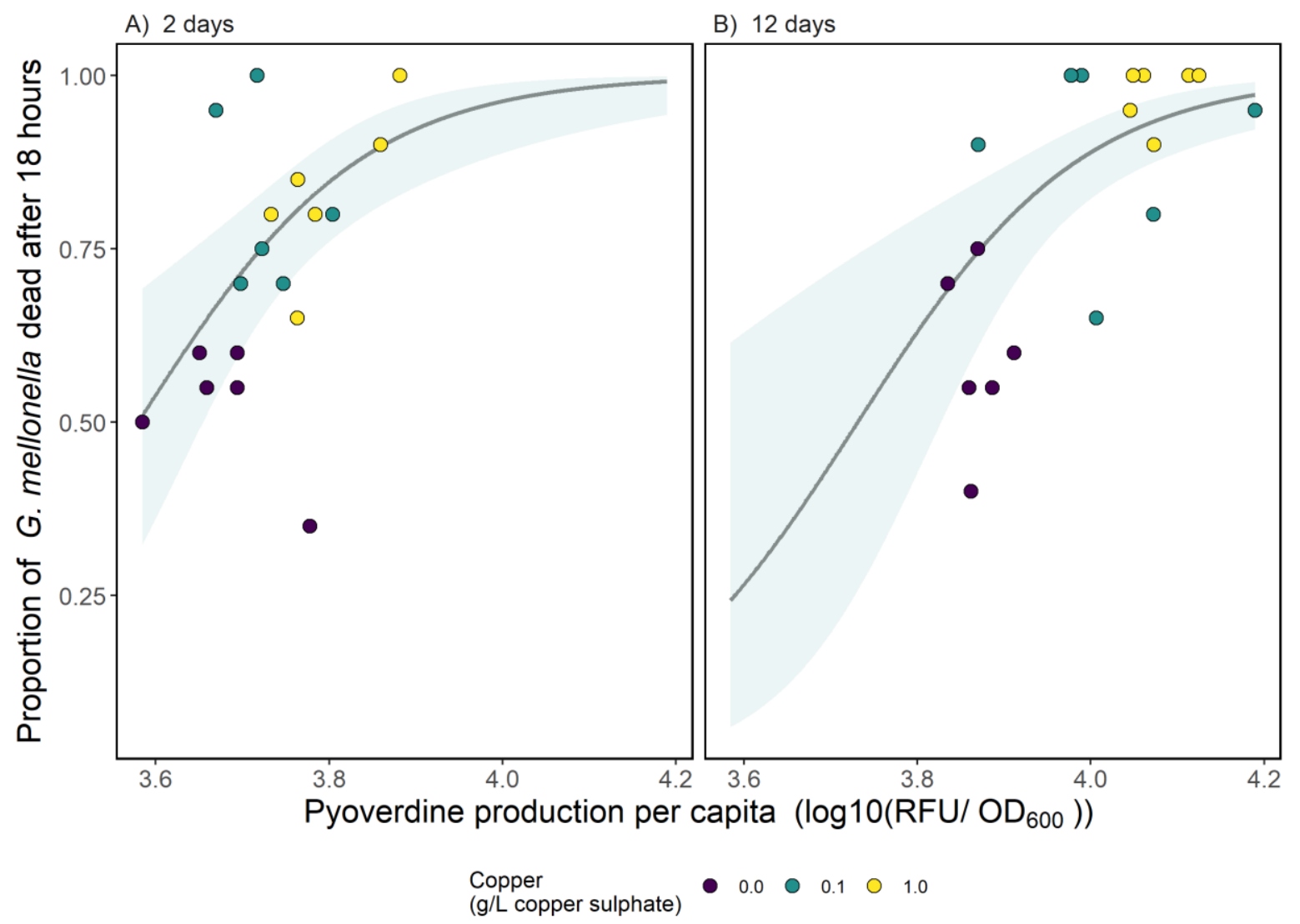

254 Figure 5 The relationship between per capita pyoverdine production $\left(\log _{10}\right.$-transformed standardised fluorescence units per $\mathrm{OD}_{600}$ ) and virulence of $P$. aeruginosa populations. Virulence was quantified using the Galleria mellonella infection model and expressed as the proportion of G. mellonella dead (out of 20) 18 hours after injection. Individual replicates are represented by circles $(n=$ six per treatment); purple points represent the control (no copper) treatments, blue the low copper and yellow the high copper treatment. The line shows the best model fit and the shaded area shows the $95 \%$ confidence interval. 
265 Here, we experimentally tested whether copper stress causes the evolution of increased

266

267

268

269

270

271

272

273

274

275

276

277

278

279

280

281

282

283

284

285

286

287

288

289

290

291

292

293

294

295

296

297 siderophore-mediated virulence in Pseudomonas aeruginosa. We found copper to select for increased per capita pyoverdine production, which resulted in greater levels of death in the Galleria mellonella infection assay. Moreover, pyoverdine production was found to increase with time such that populations exposed to copper for twelve days were more virulent than those exposed for two days. In addition to this overall increase in virulence with time, high copper stress caused significantly greater virulence than low copper stress in the twelve day treatment but not in the two day treatment. As a result the twelve-day high copper treatment demonstrated the greatest virulence. This shows that copper stress increases pyoverdine production and consequently virulence, and that this effect increases with exposure time and copper concentration.

Finding copper-mediated increases in pyoverdine production is consistent with a role of pyoverdine in detoxification of copper in P. aeruginosa populations (45). Here we show that a prolonged need for copper detoxification leads to the continued evolution of greater siderophore production. The resulting cost of these genotypic changes to a metabolically costly trait is a likely explanation for the reduced densities in the twelve-day copper treatments. Apart from copper, pyoverdine production has been shown to be up-regulated in response to $\mathrm{Al}^{3+}$, $\mathrm{Ga}^{3+}, \mathrm{Mn}^{2+}$ and $\mathrm{Ni}^{2+}(45)$. This suggests that these metals may also increase P. aeruginosa virulence, and may cause selection for increased production over longer exposure periods. Furthermore, it is likely that mixtures of metals, which are frequently encountered in polluted environments, might additively select for pyoverdine production and thereby virulence.

The positive association between pyoverdine production and virulence is consistent with previous findings, including in murine models (35). As well as directly aiding pathogen growth within a host by increasing iron uptake, pyoverdine production can increase virulence by causing the upregulation of additional virulence factors such as Exotoxin A and PrpL protease $(46,47)$. Finding per capita pyoverdine production to be a better predictor of virulence than population density in this system shows the cost of evolving greater production on density is inconsequential in a host. We note that in addition to pyoverdine production, copper stress has been shown to change the expression of over 300 genes in P. aeruginosa (48), and it is plausible these also have implications for virulence. 
In addition to $P$. aeruginosa, toxic metals have also been shown to induce the production of (non-pyoverdine) siderophores in other species (10), with siderophore production being shown to increase proportionally with toxic metal pollution in natural microbial communities (9). As the copper concentrations used in this study were chosen for their environmental relevance, we suggest our findings could be relevant to natural communities in polluted environments. We note that remediation techniques, principally lime addition, are used in some metal-polluted areas to reduce their toxic effect (49), and this can lower community siderophore production (50). Extending experiments such as those described here to the level of natural communities could shed light on the consequences of metal pollution and metal remediation on bacterial siderophore production and virulence.

In conclusion, we experimentally show that copper stress increases the virulence of the pathogen Pseudomonas aeruginosa by selecting for increased production of a metaldetoxifying siderophore. We therefore demonstrate a direct link between toxic metal stress and virulence in an opportunistic pathogen of significant clinical importance. Furthermore, we show that the effect of metal exposure on virulence increases with exposure time and copper concentration. This raises further concern for the effect of ever-increasing metal pollution on bacterial pathogens, and highlights further work is needed to understand the role of metals in bacterial virulence.

\section{Funding}

This work was supported by NERC FRESH GW4 award no. NE/R011524/1.

\section{$\underline{\text { References }}$}

1. Cuív PÓ, Clarke P, O'Connell M. Identification and characterization of an ironregulated gene, chtA, required for the utilization of the xenosiderophores aerobactin, rhizobactin 1021 and schizokinen by Pseudomonas aeruginosa. Microbiology. 2006;152(4):945-54.

2. Rajkumar M, Ae N, Prasad MNV, Freitas H. Potential of siderophore-producing bacteria for improving heavy metal phytoextraction. Trends in biotechnology. 2010;28(3):142-9.

331 3. Braud A, Geoffroy V, Hoegy F, Mislin G, Schalk I. The siderophores pyoverdine and pyochelin are involved in Pseudomonas aeruginosa resistance against metals: another 

cooperative behaviour in bacteria. Journal of evolutionary biology. 2009;22(3):589-98. 5. Winkelmann G. Ecology of siderophores with special reference to the fungi. Biometals. 2007;20(3-4):379. 6. Cassat JE, Skaar EP. Iron in infection and immunity. Cell host \& microbe. 2013;13(5):509-19.

7. Palmer LD, Skaar EP. Transition metals and virulence in bacteria. Annual review of genetics. 2016;50:67-91.

8. O'Brien S, Hodgson DJ, Buckling A. Social evolution of toxic metal bioremediation in Pseudomonas aeruginosa. Proceedings of the Royal Society B: Biological Sciences. 2014;281(1787):20140858. selection of siderophore-producing microbial taxa in response to heavy metal contamination. Ecology letters. 2018;21(1):117-27.

348 10. Saha R, Saha N, Donofrio RS, Bestervelt LL. Microbial siderophores: a mini review. Journal of basic microbiology. 2013;53(4):303-17.

11. Hider RC, Kong X. Chemistry and biology of siderophores. Natural product reports. 2010;27(5):637-57.

12. Griffin AS, West SA, Buckling A. Cooperation and competition in pathogenic bacteria. Nature. 2004;430(7003):1024-7.

13. Skaar EP. The battle for iron between bacterial pathogens and their vertebrate hosts. PLoS pathogens. 2010;6(8):e1000949. transferrin 1 mediates nutritional immunity in Drosophila melanogaster. Proceedings of the National Academy of Sciences. 2020;117(13):7317-25.

15. Golonka R, San Yeoh B, Vijay-Kumar M. The iron tug-of-war between bacterial siderophores and innate immunity. Journal of innate immunity. 2019;11(3):249-62.

16. Granato E, Harrison F, Kümmerli R, Ross-Gillespie A. Do Bacterial "Virulence Factors" Always Increase Virulence. A Meta-Analysis of Pyoverdine Production in Pseudomonas aeruginosa As a Test Case Front Microbiol. 2016;7:1952. virulence loci encoding aerobactin and salmochelin siderophore synthesis in Klebsiella pneumoniae. Genome medicine. 2018;10(1):77.

367 18. Saleem M, Shah M. Detection of Siderophore production in Uropathogenic Escherichia. coli in patients with Type 2 Diabetes Mellitus. Int J Med Microbiol Trop Dis. 2017;3:176-7.

19. Abergel RJ, Wilson MK, Arceneaux JE, Hoette TM, Strong RK, Byers BR, et al. Anthrax pathogen evades the mammalian immune system through stealth siderophore production. Proceedings of the National Academy of Sciences. 2006;103(49):18499-503. 20. Perry WJ, Spraggins JM, Sheldon JR, Grunenwald CM, Heinrichs DE, Cassat JE, et al. Staphylococcus aureus exhibits heterogeneous siderophore production within the vertebrate host. Proceedings of the National Academy of Sciences. 2019;116(44):21980-2. 21. Braud A, Geoffroy V, Hoegy F, Mislin GL, Schalk IJ. Presence of the siderophores pyoverdine and pyochelin in the extracellular medium reduces toxic metal accumulation in Pseudomonas aeruginosa and increases bacterial metal tolerance. Environmental Microbiology Reports. 2010;2(3):419-25.

22. Schalk IJ, Hannauer M, Braud A. New roles for bacterial siderophores in metal transport and tolerance. Environmental microbiology. 2011;13(11):2844-54. 
23. Höfte M, Buysens S, Koedam N, Cornelis P. Zinc affects siderophore-mediated high affinity iron uptake systems in the rhizosphere Pseudomonas aeruginosa 7NSK2. Biometals. 1993;6(2):85-91.

24. Lhospice S, Gomez NO, Ouerdane L, Brutesco C, Ghssein G, Hajjar C, et al.

Pseudomonas aeruginosa zinc uptake in chelating environment is primarily mediated by the metallophore pseudopaline. Scientific reports. 2017;7(1):1-10. 25. Harrison F, Paul J, Massey RC, Buckling A. Interspecific competition and siderophore-mediated cooperation in Pseudomonas aeruginosa. The ISME journal. 2008;2(1):49-55.

26. Dumas Z, Kümmerli R. Cost of cooperation rules selection for cheats in bacterial metapopulations. Journal of evolutionary biology. 2012;25(3):473-84.

27. Kümmerli R, Jiricny N, Clarke LS, West SA, Griffin AS. Phenotypic plasticity of a cooperative behaviour in bacteria. Journal of evolutionary biology. 2009;22(3):589-98. 28. Meyer J-M, Neely A, Stintzi A, Georges C, Holder IA. Pyoverdin is essential for virulence of Pseudomonas aeruginosa. Infection and immunity. 1996;64(2):518-23. 29. Buckling A, Harrison F, Vos M, Brockhurst MA, Gardner A, West SA, et al. Siderophore-mediated cooperation and virulence in Pseudomonas aeruginosa. FEMS microbiology ecology. 2007;62(2):135-41.

400 30. Rice LB. Federal funding for the study of antimicrobial resistance in nosocomial pathogens: no ESKAPE. The University of Chicago Press; 2008.

31. Rutherford V, Yom K, Ozer EA, Pura O, Hughes A, Murphy KR, et al. Environmental reservoirs for exoS+ and exoU+ strains of Pseudomonas aeruginosa. Environmental microbiology reports. 2018;10(4):485-92.

32. Roy-Burman A, Savel RH, Racine S, Swanson BL, Revadigar NS, Fujimoto J, et al. Type III protein secretion is associated with death in lower respiratory and systemic Pseudomonas aeruginosa infections. The Journal of infectious diseases. 2001;183(12):176774.

33. Japoni A, Farshad S, Alborzi A. Pseudomonas aeruginosa: burn infection, treatment and antibacterial resistance. Iranian Red Crescent Medical Journal. 2009;11(3):244-53.

34. Fergie JE, Shema SJ, Lott L, Crawford R, Patrick CC. Pseudomonas aeruginosa bacteremia in immunocompromised children: analysis of factors associated with a poor outcome. Clinical infectious diseases. 1994;18(3):390-4.

35. Kang D, Revtovich AV, Chen Q, Shah KN, Cannon CL, Kirienko NV. Pyoverdinedependent virulence of Pseudomonas aeruginosa isolates from cystic fibrosis patients. Frontiers in microbiology. 2019;10:2048.

36. Harrison F, Buckling A. Cooperative production of siderophores by Pseudomonas aeruginosa. Front Biosci. 2009;14:4113-26.

37. Stilwell P, O'Brien S, Hesse E, Lowe C, Gardner A, Buckling A. Resource heterogeneity and the evolution of public goods cooperation. Evolution letters. 2020;4(2):155-63.

38. Brun L, Maillet J, Richarte J, Herrmann P, Remy J. Relationships between extractable copper, soil properties and copper uptake by wild plants in vineyard soils. Environmental pollution. 1998;102(2-3):151-61.

425 39. O'Brien S, Luján AM, Paterson S, Cant MA, Buckling A. Adaptation to public goods 426 cheats in Pseudomonas aeruginosa. Proceedings of the Royal Society B: Biological Sciences. 2017;284(1859):20171089.

40. Harrison F, Browning LE, Vos M, Buckling A. Cooperation and virulence in acute Pseudomonas aeruginosainfections. BMC biology. 2006;4(1):21. 
41. Hernandez RJ, Hesse E, Dowling AJ, Coyle NM, Feil EJ, Gaze WH, et al. Using the wax moth larva Galleria mellonella infection model to detect emerging bacterial pathogens. PeerJ. 2019; 7:e6150-e. 42. Hartig F. DHARMa: Residual Diagnostics for Hierarchical (Multi-Level / Mixed) Regression Models. R package version 0422021.

43. Team RC. R: A language and environment for statistical computing. R Foundation for Statistical Computing.

44. Douglas Bates MM, Ben Bolker, Steve Walker. Fitting Linear Mixed-Effects Models Using lme4. Journal of Statistical Software. 2015;67(1):1-48.

45. Braud A, Hoegy F, Jezequel K, Lebeau T, Schalk IJ. New insights into the metal specificity of the Pseudomonas aeruginosa pyoverdine-iron uptake pathway. Environmental microbiology. 2009;11(5):1079-91.

46. Lamont IL, Beare PA, Ochsner U, Vasil AI, Vasil ML. Siderophore-mediated signaling regulates virulence factor production in Pseudomonas aeruginosa. Proceedings of the National Academy of Sciences. 2002;99(10):7072-7.

47. Lopez-Medina E, Fan D, Coughlin LA, Ho EX, Lamont IL, Reimmann C, et al. Candida albicans inhibits Pseudomonas aeruginosa virulence through suppression of pyochelin and pyoverdine biosynthesis. PLoS Pathog. 2015;11(8):e1005129.

48. Teitzel GM, Geddie A, Susan K, Kirisits MJ, Whiteley M, Parsek MR. Survival and growth in the presence of elevated copper: transcriptional profiling of copper-stressed Pseudomonas aeruginosa. Journal of bacteriology. 2006;188(20):7242.

49. Palansooriya KN, Shaheen SM, Chen SS, Tsang DCW, Hashimoto Y, Hou D, et al. Soil amendments for immobilization of potentially toxic elements in contaminated soils: A critical review. Environment international. 2020;134:105046.

50. Hesse E, Padfield D, Bayer F, Van Veen EM, Bryan CG, Buckling A. Anthropogenic remediation of heavy metals selects against natural microbial remediation. Proceedings of the Royal Society B. 2019;286(1905):20190804.

51. Seiler C, Berendonk T. Heavy metal driven co-selection of antibiotic resistance in soil and water bodies impacted by agriculture and aquaculture. Frontiers in Microbiology. 2012;3(399).

52. Paul D. Research on heavy metal pollution of river Ganga: A review. Annals of Agrarian Science. 2017;15(2):278-86.

53. Chou C, Haya K, Paon L, Burridge L, Moffatt J. Aquaculture-related trace metals in sediments and lobsters and relevance to environmental monitoring program ratings for nearfield effects. Marine Pollution Bulletin. 2002;44(11):1259-68.

54. Berg J, Tom-Petersen A, Nybroe O. Copper amendment of agricultural soil selects for bacterial antibiotic resistance in the field. Letters in Applied Microbiology. 2005;40(2):14651.

55. Bruins MR, Kapil S, Oehme FW. Microbial resistance to metals in the environment. Ecotoxicology and environmental safety. 2000;45(3):198-207.

56. Giller KE, Witter E, McGrath SP. Toxicity of heavy metals to microorganisms and microbial processes in agricultural soils: a review. Soil biology and biochemistry. 1998;30(10-11):1389-414.

57. Makvandi P, Wang Cy, Zare EN, Borzacchiello A, Niu Ln, Tay FR. Metal-based nanomaterials in biomedical applications: Antimicrobial activity and cytotoxicity aspects. Advanced Functional Materials. 2020:1910021.

58. Vincent M, Hartemann P, Engels-Deutsch M. Antimicrobial applications of copper. International journal of hygiene and environmental health. 2016;219(7):585-91. 